\title{
EGFR NP_005219.2:p.L747del
}

National Cancer Institute

\section{Source}

National Cancer Institute. EGFR NP 005219.2:p.L747del. NCI Thesaurus. Code C98565.

A deletion of the leucine at position 747 from the epidermal growth factor receptor protein. 